\title{
PLCG2-associated antibody deficiency and immune dysregulation
}

INSERM

\section{Source}

INSERM. (1999). Orphanet: an online rare disease and orphan drug data base. PLCG2associated antibody deficiency and immune dysregulation. ORPHA:300359

PLCG2-associated antibody deficiency and immune dysregulation is a rare, hereditary, immune deficiency with skin involvement characterized by early-onset cold urticaria after generalized exposure to cold air or evaporative cooling and not after contact with cold objects. Additional immunologic abnormalities are often present - antibody deficiency, recurrent infections, autoimmune disease and symptomatic allergic disease. 